\title{
A PROJECTION GRAPHIC DISPLAY FOR THE COMPLTER AIDED ANALYSIS OF BUBBLE CHAMBER IMAGES
}

\author{
E. SOLOMOS \\ CERN, Genera, Siritzerland
}

Received 4 September 1979

\begin{abstract}
A projection graphic display for aiding the analysis of bubble chanber photographs has been developed by the Instrumentation (iroup of EF Division at CERN.

The display image is generated on a very high brightness cathode ray tube and projected on to the tathe af the scanning-measuring machines as a superposition to the image of the bubble chamber. The display (wn send messiges in the operator and aid the measurement by indicating directly on the chamber inage the tracks which are measured cirrecily or not.
\end{abstract}

\section{Introduction}

The idea of projecting a computer generated display on to the bubble chamber film scanning and measuring tables is not new. However, the important technical problems related to it did not permit the realization of such a project.

There exist several technologies for television image projection, but all of them cost an order of magnitude more than the admissible cost for a scanning-measuring machine display, which cannot be more than about ten thousand dollars. The projection display cost could come down only by the elimination of all the unnecessary features of a standard television projector and by adaptation of the system to the specific requirements of the bubble chamber film analysis.

Such a system has been developed and installed on one of the Bessymatic tables. The display image is generated on a very high brightness cathode ray tube (CRT) and projected, via an optical system, on to the table, as an optical overlay to the projected image of the bubble chamber photo.

The performances of the system are satisfactory and should encourage a further development and optimization of the existing prototype.

\section{Why a projection display?}

Most of the qualitative analysis and sometimes the precision measurement of the bubble chamber images is performed by a skilled operator on a projection table. An on-line computer gathers the information and aids the operator's action. Very often the computer must indicate to the operator various parts of the image; as for example fiducial marks, vertices or tracks. On the Bessymatic table this is performed at present by sending part of the image to a standard television camera and displaying it on a monitor together with the computer generated display.

A direct projection of the graphic image on the scanning-measuring table has the following advantages in comparison to the method outlined above:

a) The operator always examines the same image. Therefore, the probability of errors, as for example the confusion of similar patterns. will be less.

b) A light-pen system can be used with the table whenever fast low-accuracy predigitizings are required. The same light pen could eliminate the operator's keyboard.

\section{A choice between various principles of operation}

Before the detailed description of the operation and the problems of such a display there follows a short review of all existing television projection technologies. The reasons which led to the choice of the above method will be argued.

In principle there are three general methods for projecting a television (or computer display) image: (a) by deflection of a light beam: (b) using light valves and (c) by projection of a bright CRT imagc. These three methods are now examined in more detail $^{1.2}$ ).

\subsection{LIGHT BFAM DEFIFCTION}

The screen is illuminated by a light beam (usually a laser) whose intensity is modulated by the video signal. The light spot is moved anywhere on the screen by deflecting the beam electromechanically or electro-optically. The electromechanical de- 
flection is not fast enough if a complex, randomly scanned pattern is required. Alternatively the electro-optical methods, which employ sets of polarizing crystals, have a very low light transfer coefficient and low resolution. In addition to this, the modulation of a powerful laser beam is not easily realizable. Such systems are still in the experimental stage and none of them have been commercially used.

\subsection{Light VALVES}

The projection of a variable transparency image, in the same way as a photographic slide, is the most elegant and straightforward way to form large screen images. The advantages of these systems which are called "light valves" is that the light intensity is not directly related to the original image. The required optics are also rather simple.

In spite of the great difficulties which present the realization of rapidly addressable and erasable light valves, the most successful large screen projection displays are based on this method ${ }^{3-5}$ ).

The most widely used light valves employ "schlieren optics" in combination with deformable surfaces (oil-films, plastics or microscopic mirror matrices), which are deformed owing to electric charge influence. The charge is deposited by a deflectable electron beam.

Other valves use "Pockels-effect" materials in combination with polarizers. Photochromic materials which change transparency under ultraviolet exposure are also used. The "liquid-crystal" matrix is also available but it still presents very low switching times. All these methods employ an electron beam for addressing a point on the valve surface where they either create a local potential or they produce a secondary ultraviolet emission.

Although the light valves are very successful in large screen applications, their cost remains an order of magnitude higher in comparison to good quality direct view displays. Moreover, a randomly scanned pattern is not easily implemented, especially if this pattern is changing. Furthermore, before each image is drawn, the previous one has to be erased. This tends to cause flickering of the display.

\subsection{DiRECT PROJECTION OF A BRIGHT CRT}

The cathode ray tube (CRT) has been exploited for a long time in television applications. The techniques to obtain a very bright image while maintaining a good resolution have been well known for the past forty years. Recently new high efficiency phosphor types with a variety of colours and persistences have been developed. In addition to this, owing to the enormous market of television apparatus, standard electronic driving components can be obtained at low prices ${ }^{6,7}$ ).

The formation of randomly scanned images on a CRT presents no problems. In comparison to raster scanning, random scanning offers better resolution and line quality at much lower deflection and video bandwidths. This type of scanning is perfectly

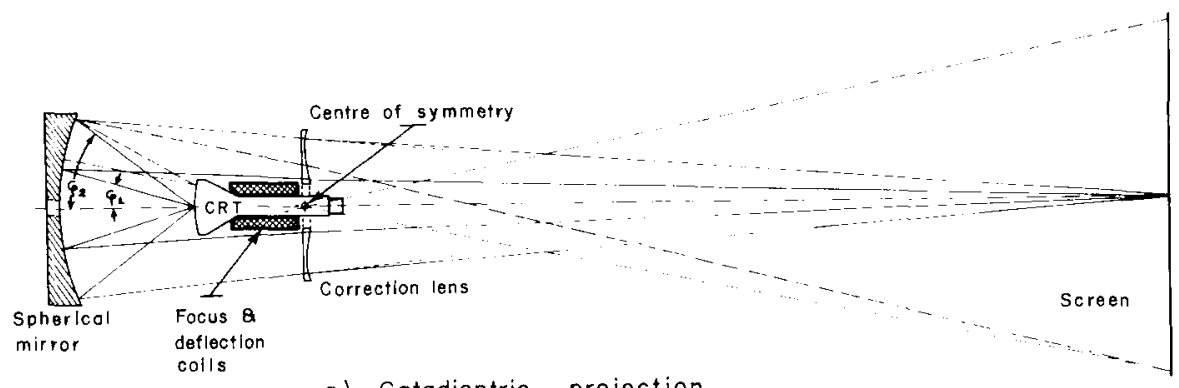

a) Catadioptric projection

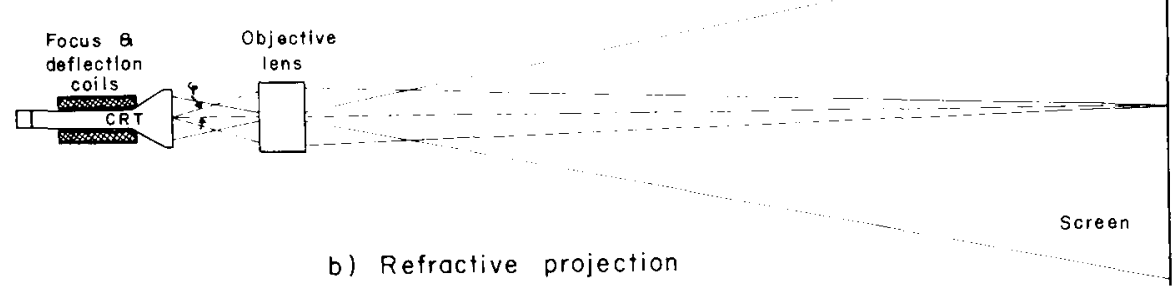

Fig. 1. The two basic principles for the optics used to project a bright CRT image. 
adapted to the generation of graphic images by means of a set of linear vectors.

The high brightness with good resolution is obtained by raising the electron acceleration voltage. In current applications, the anode voltage of high brightness CRTs varies between 30 and $60 \mathrm{kV}$, giving typical values of brightness from 5000 to $20000 \mathrm{~cd} / \mathrm{m}^{2}$. Peak values of brightness can be even higher, but it depends strongly on the duration of the bright intervals and the method of dissipation of the produced heat.

The direct projection of the CRT image is obtained via a large aperture optical system; usually a catadioptric "Schmidt" projector ${ }^{8}$ ). In such systems the optics form the greatest part of the total system cost. In applications where a lower magnification and projection distance are possible, the use of standard refractive objectives becomes more economic (fig. 1).

\section{The choice of the optimum optical system}

The choice of the optical system for a projection display cannot be made without taking into consideration the desired display characteristics as well as technical and economic constraints. Once all these factors have been defined one can vary the parameters to obtain an optimum system. Therefore, there exists no optimum optics independently of the total display system.

For the specific application of aiding the bubble chamber picture analysis, the display characteristics could be listed as follows:

(a) image size;

(b) line thickness;

(c) image brightness;

(d) colour.

As far as the technical constraints are concerned, one can fix minimum or maximum values for the above basic display parameters. It is obvious that these limits are related. For example, if the projected image size has to be increased while its relative resolution and illumination are maintained at their maximum limits, it is necessary to accept a larger projected line thickness and higher brightness on the CRT faceplate.

In addition to these constraints, other important technical factors have to be met:

(e) maximum trace length of the randomly scanned image;

(f) projector compactness;

(g) projection distance.
The last two factors are very important in the present application where the display image has to be superimposed on to a usual photographic image. This is because the one projector should not obstruct the light path of the other projector. Consequently, if a long projection distance is needed, the physical aperture of the optical system must be larger. If on the contrary, a lower projection distance is chosen, a skew projection is necessary, which means that a larger angle optical system must be employed (fig. 2).

The maximum display trace length is directly proportional to the deflection speed and inversely proportional to the display refresh rate. If the deflection speed is kept to a minimum, the consumed deflection power is considerably cut down. The cost of power supplies, deflection amplifiers, video drivers and ventilation is also kept at a minimum. This cost is even more important in high brightness displays where, owing to the higher acceleration voltage, a greater amount of current is needed to deflect the beam electrons.

The question arises: should one use catadioptric or refractive optics? The principle is that the larger amount of light the optical system collects, the

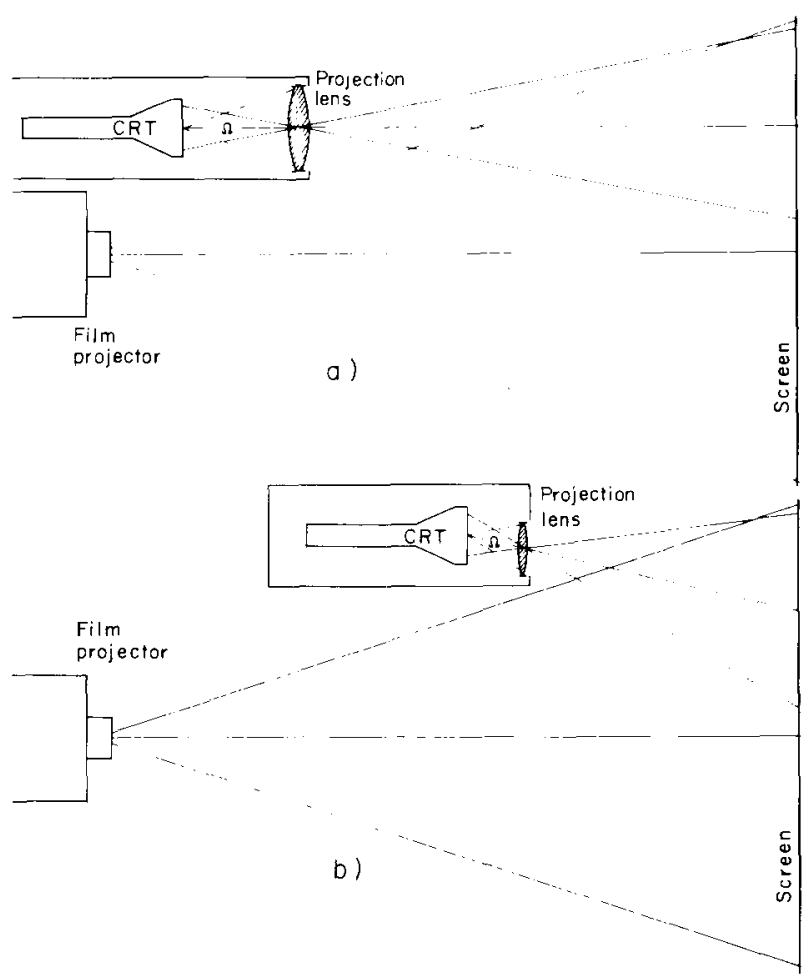

Fig. 2. The influence of the projection distance to physical aperture and field angle of the projection objective lens. 
better it is. However, a standard refractive photographic objective can cost about ten times less than a non-standard optical system. Therefore, if a high aperture commercially available optical system can produce the required image, one must choose this standard product. In the present case it was found that an aerophotography refractive objective could cover very well the field of a $12 \mathrm{~cm}$ diameter CRT at a focal length of 25 to $30 \mathrm{~cm}$. Similar objectives but with insufficient resolution have been produced specially for consumer television projection applications. Recently a big television manufacturer announced the production of a home TV-projector using the same principle but employing cheaper moulded plastic objectives.

Having chosen a display image size of $60 \mathrm{~cm}$, the magnification is $m=5$. The ratio between the image illumination $E$ and the CRT brightness $L$ is found by the following formula ${ }^{9-11}$ ):

$\frac{E}{L} \simeq \frac{\pi T}{4 f^{2}(m+1)^{2}}$,

where $T$ is the total transfer coefficient of the optical system and $f^{t}$ is the focal length to aperture ratio.

Applying $T=0.9, f^{*}=2.5$ and $m=5$, a value for $E / L=320$ is found, which means that for each lux of illumination of the projected image, the CRT faceplate brightness must be $320 \mathrm{~cd} / \mathrm{m}^{2}$.

After certain simulation tests it was found that ihe optimum characteristics for a display to be superimposed on a bubble chamber image must be as follows:

(a) illumination equal or several times greater than the average illumination of the bubble chamber image;

(b) line thickness between 0.5 and $1 \mathrm{~mm}$;

(c) a nearly saturated colour, preferably green, for which the human eye is more sensitive.

Under these conditions an illumination between 20 and 30 lux at a resolution of 600 to 1000 lines corresponds to a tube brightness of 6500 to $9600 \mathrm{~cd} / \mathrm{m}^{2}$. This fairly high brightness-resolution is easily obtained with a standard television projection tube operating at $50 \mathrm{kV}$ and having magnetic focusing.

The alternative of using a catadioptric system should not be excluded if more than a few projectors have to be produced. In this case, an aperture of up to $\mathrm{f}^{\prime \prime}=1.3$ with a $30 \mathrm{~cm}$ diameter spherical mirror is feasible. The Schmidt correction plate could be replaced by a cheaper "Maksutov" meniscus $^{12}$ ). A preliminary simulation study carried out by the author in collaboration with optical engineers of the European Southern Observatory, showed that a catadioptric system corrected by such a meniscus could project the image without significant loss of its original resolution ${ }^{13}$ ).

Very often the projection tubes found in the market have already a spherical face-plate calculated for certain catadioptric Schmidt systems. The eventual curvature of the tube face can be optically modified by means of a simple lens put in front of it. On the developed phototype, the tube has a spherical face with $207 \mathrm{~mm}$ of radius. This curvature has been corrected by means of a bi-concave lens with radii $113.5 \mathrm{~mm}$ and $217.2 \mathrm{~mm}$. The biconcave lens introduces certain geometrical abberations which degrade slightly the quality on the outer limits of the image. A barrel distortion which is also introduced is not a problem as it can be easily corrected electronically.

\section{The electronic circuit}

The block diagram of the electronic circuit is shown in fig. 3. The only difference from a usual CRT display is the value of the acceleration voltage and deflection currents, as well as the existence of a special "Security Unit". With an anode voltage of $50 \mathrm{kV}$, a pair of $30 \mu \mathrm{H}$ deflection coils need about $\pm 10 \mathrm{~A}$ of current for full deflection. The spot settling time $(99 \%$ of a step movement) is around $10 \mu \mathrm{s}$ with an amplifier voltage of $\pm 45 \mathrm{~V}$. Operat-

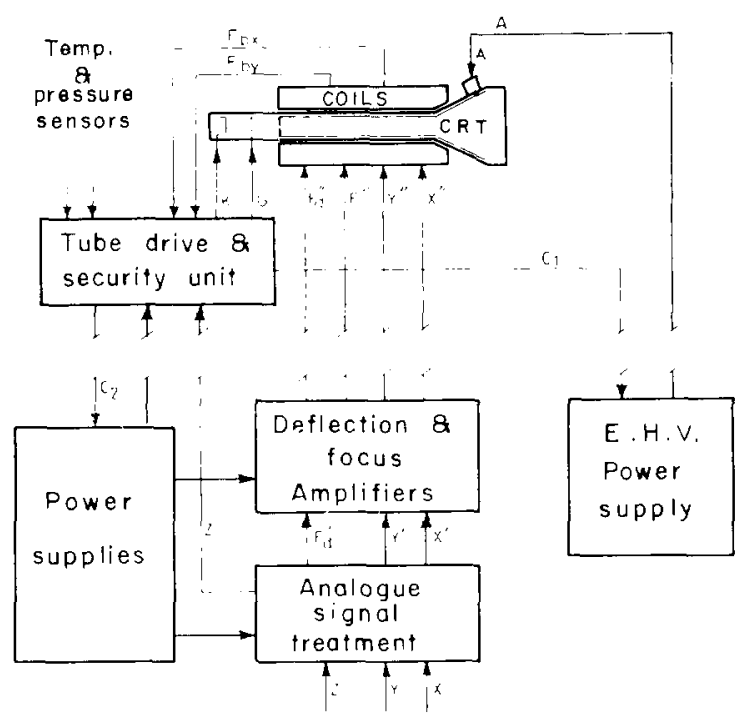

Fig. 3. The block diagram of the electronic circuit. 


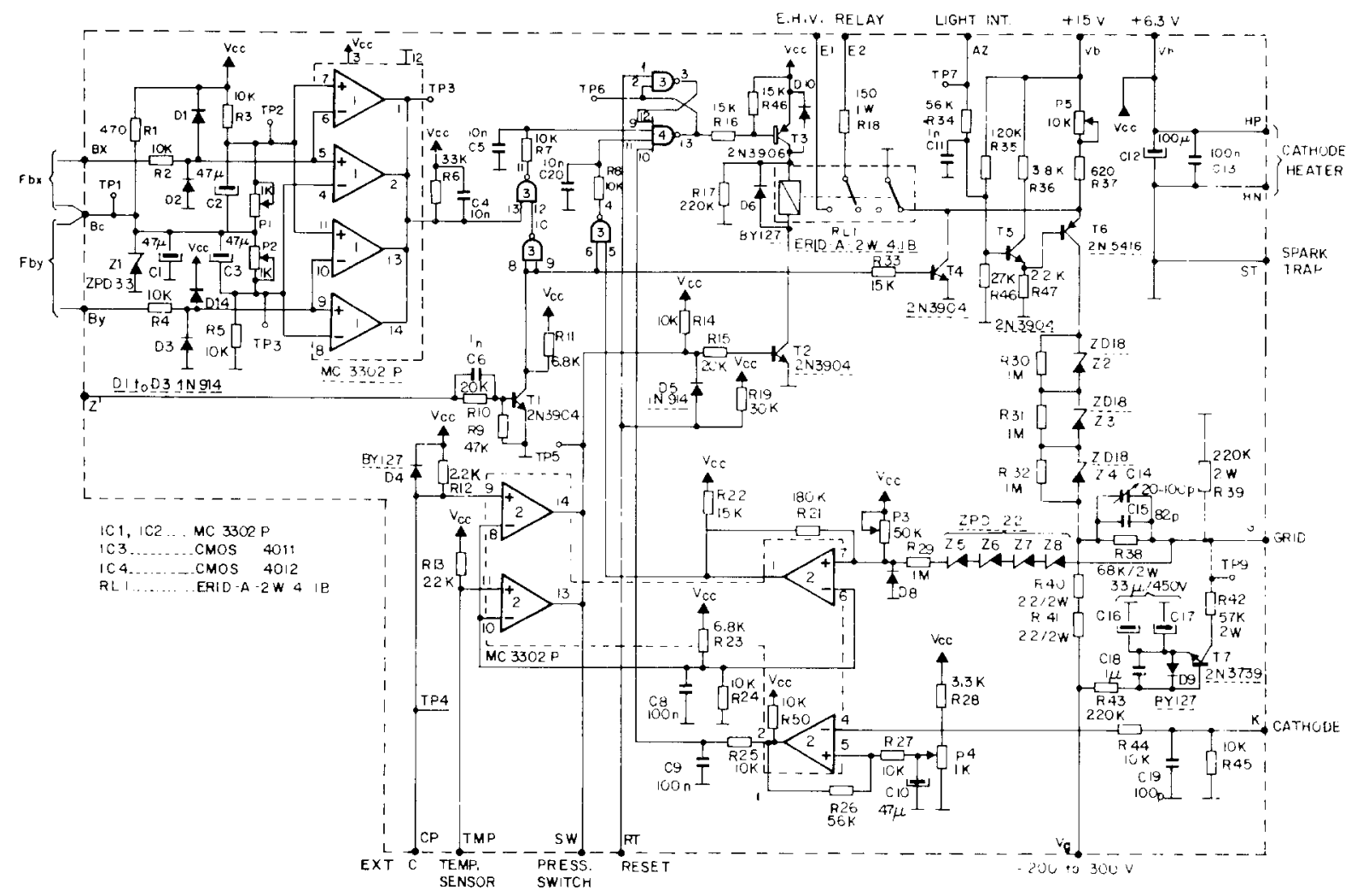

Fig. 4. The security unit and grid driver.

ing the amplifier at lower supply voltages increases the settling time, but this still remains sufficient for the rather simple graphic patterns needed in the bubble chamber film analysis. The tube. the focusdeflection coils and the amplifiers are manufactured by Ferranti Ltd., (new name Thorn Brimar Ltd..) and carry the component type number $13 \mathrm{I} / 00$, DE2051 and SA 102, respectively.

The cathode ray tube current is between 80 and $100 \mu \mathrm{A}$ which implies a beam power of 4 to $5 \mathrm{~W}$. This highly focused energy can easily burn the phosphor or even melt the tube glass if the spot remains stationary. Therefore, a special security unit is needed to cut-off the high voltage on a stationary deflection while the beam current is on. Another dangerous condition for the tube is the presence of a high voltage with a cold cathode, a combination which could produce a cold electron extraction and destroy the cathode coating. The security unit guarantees that at a turn-on action the high voltage will be applied at least two minutes after the heating voltage. Moreover, at a turn-off action a negative cut-off voltage is applied to the control grid during the high voltage discharge period.

A more detailed drawing of the security unit and the grid driver is shown in fig. 4. Four voltage comparators (IC1) detect the stationary deflection condition by testing the voltage output of two feedback coils which are closely coupled to the deflection coils. The R-S flip-flop (TP6) which switches on the high voltage supply via a relay RLl is cleared on stationary deflection with Z-on or with high (more positive) grid voltage. The latter condition is detected by the voltage comparator $2 / 1^{*}$. The comparator $2 / 2$ detects if the beam current exceeds the maximum permitted value. The comparator $2 /$ 14 does not allow RLl to turn on, unless a $40000 \mu \mathrm{F}$ external capacitor is half way charged through R12, permitting a two minute cathode heating time. Similarly, a temperature sensor and a cooling-air activated switch could be connected to both sides of the comparator $2 / 13$ to disable the operation of the relay.

* Integrated circuit components are indicated by thesir pachige number followed by their main output lead number. 
The grid driver consists of a controlled current source T4, T5, T6 passing current through a load resistor R40, R41. The voltage on this load drives the grid via the protection resistor R38. The network around $\mathrm{T} 7$ guarantees a cut-off potential during the high voltage discharge period of a turnoff action.

\section{Problems of the system housing and screening}

The housing design of the projection display is very important because it has to fulfil certain requirements, which sometimes are mutually conflicting:

(a) insulation of the parts operating at a $50 \mathrm{kV}$ potential from the rest of the system which is around ground potential;

(b) screening of all $\mathrm{X}$-rays produced on the tube faceplate;

(c) compactness, particularly as a very short length is required along the projection axis, in order to avoid shadowing of the superimposed bubble chamber image;

(d) easy dismantling and tube change;

(e) easy optical and electrical alignment.

It was found that an optimal arrangement was the one illustrated in fig. 5. The shaded areas indicate the high voltage insulating material. The thick dark lines indicate the lead shielding for the Xrays.
The tube housing and support is composed of concentric sliding cylinders which permit the replacement of the tube with practically no adjustment. The adjustment of the optical path skewness, which in the present case is desired, is simply controlled by adjusting the position of the flat mirror.

Fig. 6 shows the projection display mounted on a Bessymatic scanning-measuring machine.

\section{Conclusion}

In spite of the important technical problems, such as optics, high voltage, high power and X-rays, a projection display on the bubble chamber analysis tables is feasible at a cost comparable to the cost of a good quality direct view CRT display. The working prototype developed in the Instrumentation Group of CERN, EF Division, projects a randomly scanned graphic image with apparent line thickness less than $1 \mathrm{~mm}$ over a circular display image of $60 \mathrm{~cm}$. The green-yellow coloured image having an illumination of 10 to 20 lux can be very easily distinguished on a superimposed bubble chamber image of the same or even ten times higher illumination. The total component cost of the prototype was about $\$ 10000$ (1978 prices), from which $\$ 1200$ was spent on the tube and another $\$ 3000$ on deflection coils and amplifiers.

A second version of the system could be

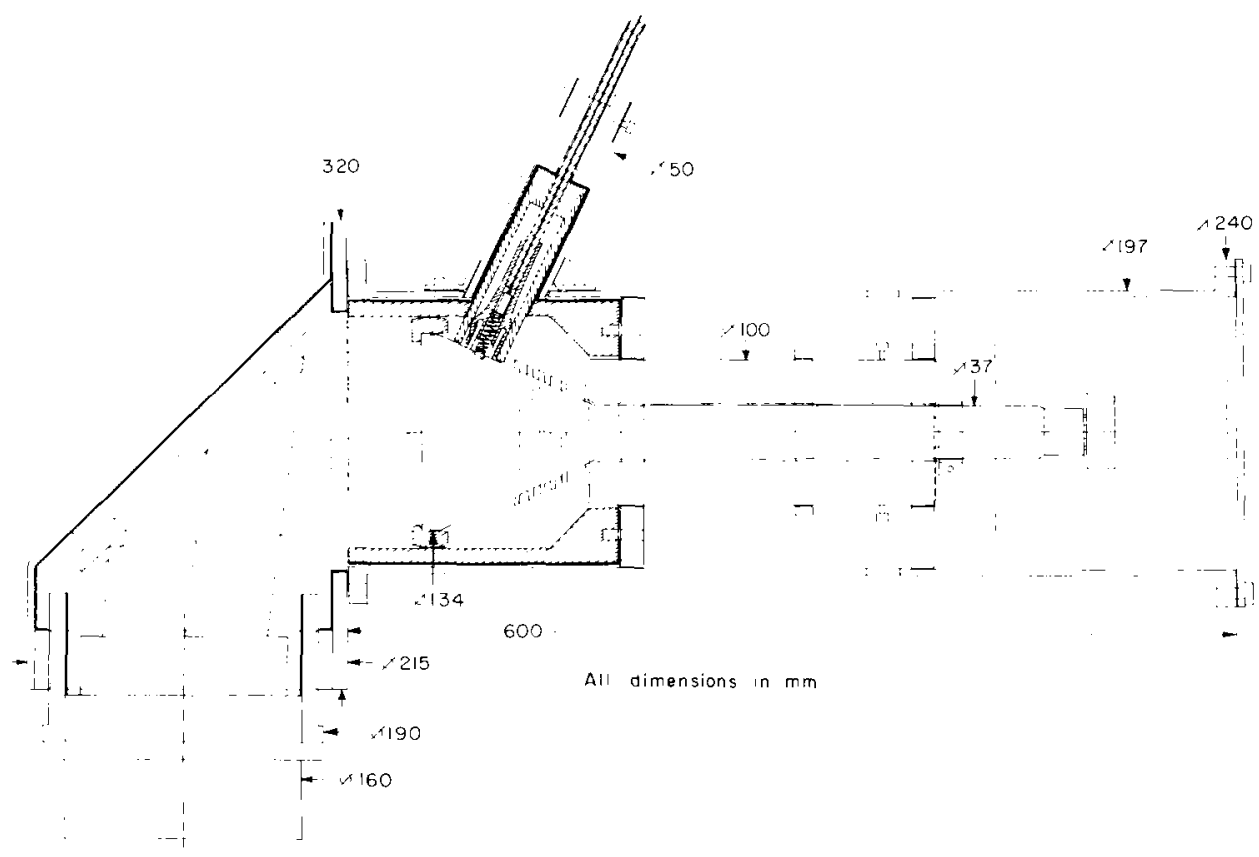

Fig. 5. Mechanical lay-out of the projection display. 


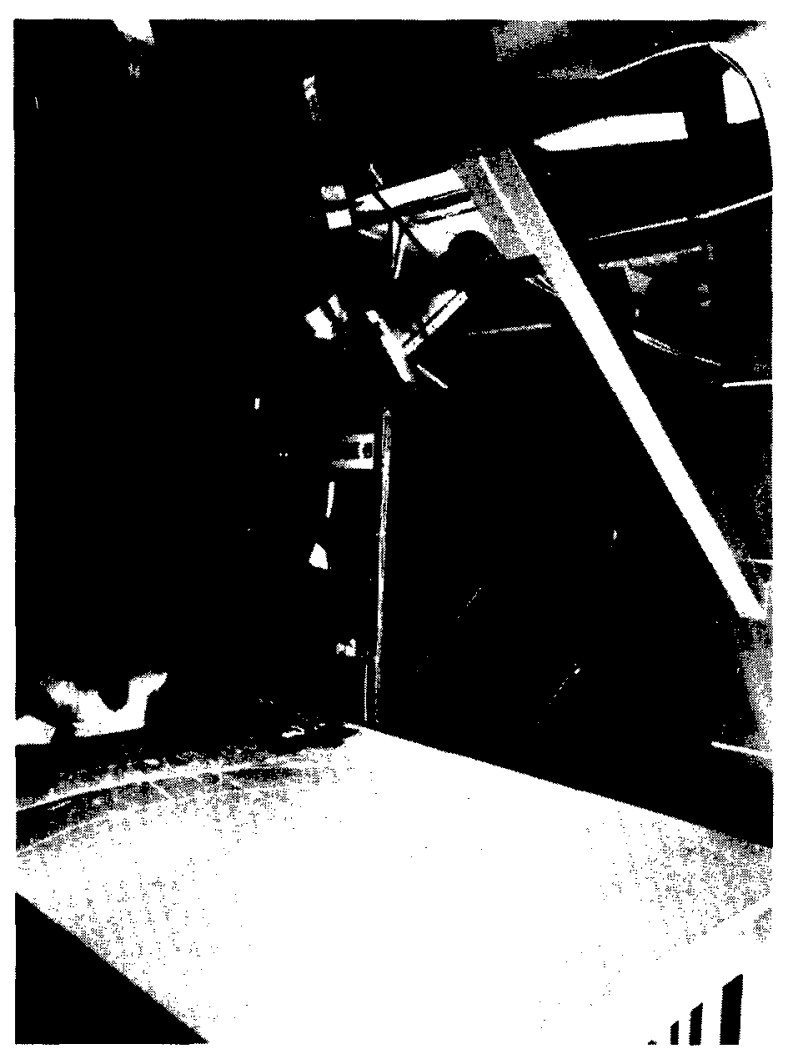

Fig. 6. The projection display mounted on a Bessymatic scanning-measuring machine.

improved from the point of view of cost. A flat face tube with dynamic focusing will produce an image with better brightness and resolution. Moreover, using a white phosphor tube at higher brightness levels together with a colour wheel* a multi colour image is easily attainable.

The author would like to thank Mr. H. Anders and Mr. H. J. Oropesa for their help with the cathode ray tube choice and alignment and $\mathrm{Mr}$. J. Dupraz for building the mechanical parts

* The "colour wheel" is an early method of colour television. The coloured image is produced by three successive blackand-white images which are projected through three corresponding primary colour filters mounted on a rotating wheel.

\section{References}

1) S. Sherr. Fundamentals of display system desizn (Wiley-Interscience, New York, 1970).

2) A Robertson, Wireless World (October 1976).

3) E. I. Sponable, J. SMPTE 60 no. 4 (April 1953).

4) J. Donjon, Lionde Ėlectrique, 58 no. 8-9 (1978)

5) R. Thomas et al., IEEE Trans. Electron Devices, ED-22 no. 9 (September 1975).

6) D.W. Epstein and I. G. Malolf, Soc. Matrix Picture Engineers 44 no. 6 (June 1945).

7) N. Patrick, Developments in cathode rav tubes for the Schmidt projector, in Pros. IEEE conlerence on display devices (October 1972).

8) I. G. Malotf and D. W. Epstein, Electronics (December 1944).

y) M. Herzberger, Modern geometrical optics (Interscience, New York, 1950).

10) R. S Longhurst, Geometrical and physical optics (Longman, New York, 1973).

11) J. Maxwell, Catadioptric imaging ststems (Adam-Helger, London, 1971).

12) D. D. Matsutov, Optical Soc. Amer. 34 no. 5 (May 1944).

13) B. Delabre and M. Le Luver, Projection of symthetic bubble chumber images. Optical data for a Maksutor twe system. Europcan Southern Observatory, Notes: IDG 77-193 (October 1977) and IDG 77-118 (December 1977) 